\title{
A Framework for Robust Synchronization in Heterogeneous Multi-Agent Networks
}

\author{
Enrico Lovisari and Ulf T. Jönsson
}

\begin{abstract}
A general framework for the analysis of networks of heterogeneous agents is presented. By modeling each agent as a nominal linear time-invariant system plus a possibly nonlinear perturbation and the interconnection among the agents via a memoryless operator, a general result is offered which ensures robust synchronization of the network to a subspace of $\mathbb{R}^{N}$. The result is applied to the case when the interconnection operator is a constant normal matrix and the perturbations nonlinear. The criterion is reduced to a graphical Popov criterion for the synchronization of the network.
\end{abstract}

\section{INTRODUCTION}

In the past few years, the scientific community has devoted a vast amount of effort to the study of synchronization in large-scale networks. In these systems a possibly huge number of agents interact according to some local law which is designed to achieve some global goal. Since the control is based on local cooperation between the agents rather than on coordination by a centralized unit, we call this a distributed algorithm. The network is modeled by a communication graph $\mathcal{G}=(V, \mathcal{E})$ in which the nodes are the agents, and an edge $(k, j)$ exists if agent $j$ is able to use some sort of information coming from agent $k$. We say that cooperation is local since each agent receives information from a usually small subset of the network, called its neighborhood. One of the simplest instances is the consensus problem, which found several applications in recent years, see [1], [2] and the references therein. It is defined as follows: Assume we have $N$ agents, each initialized with a different real number. The goal is to agree on a common value by iteratively exchanging information between the agents. We stack all these initial values in a vector $\boldsymbol{x}(0)=\boldsymbol{x}_{0} \in \mathbb{R}^{N}$, which evolves according to the discrete-time system

$$
\left\{\begin{array}{l}
\boldsymbol{x}=\frac{z^{-1}}{1-z^{-1}} \boldsymbol{u}=N_{0}\left(z^{-1}\right) \boldsymbol{u} \\
\boldsymbol{u}=-(I-P) \boldsymbol{x}
\end{array} .\right.
$$

The first equation says that agent $k$ updates its information by integration of some local input $u_{k}$, which is computed as a linear combination of the values of its neighbors with coefficients given by the $k$-th row of the matrix $P \in \mathbb{R}^{N \times N}$. This is a non-negative row-stochastic primitive ${ }^{1}$ matrix consistent with the communication graph, namely such that $P_{k j}>0$ if and only if $(j, k) \in \mathcal{E}$, in accordance to the fact

E. Lovisari is with the Department of Information Engineering, University of Padova, via Gradenigo 6/B Padova, Italy, lovisariedei.unipd.it

U.T. Jönsson is Division of Optimization and Systems Theory and the ACCESS Linneaus Centre,Royal Institute of Technology, SE 10044 Stockholm, Sweden ulf jemath.kth. se

The research leading to these results has received funding from the European Community's Seventh Framework Programme under agreement n. FP7-ICT-223866-FeedNetBack.

${ }^{1}$ Row-stochastic means $P \mathbf{1}=\mathbf{1}$, primitive that there exists a positive integer $\nu$ such that all the entries of $P^{\nu}$ are strictly positive. Here $\mathbf{1}=$ $\frac{1}{\sqrt{N}}[1, \ldots, 1] \in \mathbb{R}^{N}$. that $j$ uses, to compute its local control, only the information coming from its neighbors. By Perron-Frobenius theorem [3] we know that

$$
x_{k}(t) \stackrel{t \rightarrow \infty}{\longrightarrow} \pi^{T} \boldsymbol{x}_{0}, \forall k=1, \ldots, N
$$

where $\pi^{T} P=\pi^{T}$ and $\pi^{T} \mathbf{1}=1$.

A consensus network is thus a network of integrators that asymptotically agrees on the same linear combination of the initial conditions of the network. In this paper we are interested in higher order consensus problems, where $N_{0}\left(z^{-1}\right)$ is a generic transfer function, not just an integrator. The consensus here is required in the outputs of the agents, and we call this synchronization of the network. We consider moreover heterogeneous networks, in which the dynamics of the agents contain a nominal part perturbed in some "mild" way. The unperturbed network is called homogeneous. In [4] robust synchronization of a network of perturbed integrator is studied, and the idea is extended in [5] to the synchronization of a class of higher order systems. In [6] a graphical criterion is proposed for a homogeneous network in the case of linear dynamic feedback. The paper [7] proposes a comprehensive model for synchronization in an input/output framework. Finally, the paper [8] presents an LMI-based tool for the synchronization of systems around a given autonomous trajectory of the nominal, possibly nonlinear, system. Much effort has also been devoted to the study of the stabilization of an interconnected network (in consensus we stabilize the system apart from the consensus direction, e.g. 1), see, for example, [9], [10]. In this paper we propose a general framework for the synchronization of a network of agents. Here we allow heterogeneity, higher order dynamics and higher order synchronization, and some degree of generality in the law that produces the local controls given the local information. We present a synchronization result which is derived using the IQC theorem in [11] and we particularize it to a special case for which the criterion decomposes to one lower dimensional criterion for each eigenvalue of the interconnection matrix.

\section{Notations}

The Hilbert space $\mathcal{H}$ denotes either the continuous time signal space $\mathbf{L}_{2}[0, \infty)$ or the discrete time signal space $l_{2}(0, \infty)$. Vector valued versions are denoted by $\mathcal{H}^{n}, n$ begin the dimension of the signal. The corresponding extended space $\mathcal{H}_{e}^{n}$ consists of signals for which $P_{T} v \in \mathcal{H}^{n}, \forall T \geq 0$, where the truncation operator is defined as $\left(P_{T} v\right)(t)=v(t)$ when $t \leq T$ and $\left(P_{T} v\right)(t)=0$ when $t>T$.

An operator $H$ on $\mathcal{H}_{e}^{n}$ is causal if $P_{T} H(v)=P_{T} H\left(P_{T} v\right)$ for all $T \geq 0$ and all $v \in \mathcal{H}_{e}^{n}$. The operator is bounded if its gain $\gamma(H)=\sup _{\substack{v \in \mathcal{H}^{n} \\ v \neq 0}} \frac{\|H(v)\|}{\|v\|}$ is bounded, where $\|\cdot\|$ is the norm on $\mathcal{H}^{n} . \stackrel{v \neq 0}{H}$ is linear if $H\left(\alpha_{1} v_{1}+\alpha_{2} v_{2}\right)=$ 
$\alpha_{1} H\left(v_{1}\right)+\alpha_{2} H\left(v_{2}\right)$. A causal linear time-invariant (LTI) operator acts as

$$
\begin{aligned}
& (H v)(t)=\int_{0}^{t} h_{c}(t-\tau) v(\tau) d \tau+h_{0} v(t) \\
& (H v)(t)=\sum_{k=0}^{n} h(t-k) v(k)
\end{aligned}
$$

in continuous and discrete time, respectively, where $h$ is the impulse response function. If the signals belong to $\mathcal{H}^{n}$ then we have the equivalent frequency domain representations $\widehat{H v}(j \omega)=\widehat{H}(j \omega) \widehat{v}(j \omega)$ and $\widehat{H v}\left(e^{j \omega}\right)=\widehat{H}\left(e^{j \omega}\right) \hat{v}\left(e^{j \omega}\right)$, where $\widehat{H}$ and $\hat{v}$ is the transfer function and Fourier transform of $v \in \mathcal{H}^{n}$, respectively. $\widehat{H}(s)$ and $\widehat{H}\left(z^{-1}\right)$ will denote respectively the Laplace transform and the $Z$ transform of $h$ in continuous and discrete time. We denote by $\Omega$ the unstability domain. In the case of continuous time systems we have $\Omega=\{s: \operatorname{Re} s \geq 0\}$ and in the case of discrete time systems we have $\Omega=\{z:|z| \geq 1\}$. We let $\mathcal{H}_{d}^{n}$ denote the signals on the doubly infinite time-axis $\mathbf{L}_{2}^{n}(-\infty, \infty)$ or $l_{2}^{n}(-\infty, \infty)$ with corresponding frequency domain space $\widehat{\mathcal{H}}_{d}$ being either $\mathbf{L}_{2}(j \mathbb{R})$ or $\mathbf{L}_{2}[0,2 \pi]$. The adjoint of a bounded operator $\Psi: \mathcal{H}_{d}^{n} \rightarrow \mathcal{H}_{d}^{n}$ is defined by the relation $\langle w, \Psi v\rangle=$ $\left\langle\Psi^{*} w, v\right\rangle, \forall w, v \in \mathcal{H}_{d}^{n}$, where $\langle\cdot, \cdot\rangle$ denotes the inner product. $\Psi$ is self-adjoint if $\Psi=\Psi^{*}$. A self-adjoint bounded linear time-invariant operator $\Psi: \mathcal{H}_{d}^{n} \rightarrow \mathcal{H}_{d}^{n}$ defines a quadratic form $\langle v, \Psi v\rangle$. We say that $\Psi$ is positive definite, which is denoted, $\Psi>0$, if there exists $\epsilon>0$ such that $\langle v, \Psi v\rangle \geq \epsilon\|v\|^{2}$ for all $v \in \mathcal{H}_{d}^{n}$. A necessary and sufficent condition is that $\widehat{\Psi}(j \omega)=\widehat{\Psi}(j \omega)^{*}>0, \forall \omega \in \mathbb{R} \cup\{\infty\}$ and $\widehat{\Psi}\left(e^{j \omega}\right)=\widehat{\Psi}\left(e^{j \omega}\right)^{*}>0, \forall \omega \in[0,2 \pi]$, respectively. In this paper we use the algebra $\mathcal{A}_{c}$ consisting of LTI operators with impulse responses functions $h(t)=h_{c}(t) \theta(t)+h_{0} \delta(t)$ where $h_{c} \in \mathbf{L}_{1}^{m \times m}[0, \infty), h_{0} \in \mathbb{R}^{m \times m}, \theta(\cdot)$ and $\delta(\cdot)$ denote the unit step function and the dirac delta function, respectively. It defines a bounded LTI operator via the convolution in Eq. 2. We let $S_{\mathcal{A}_{c}}^{n \times n}$ be the bounded LTI operators on $\mathbf{L}_{2}^{n}(-\infty, \infty)$ defined by impulse response functions of the form $h(t)=$ $h_{c}(t)+h_{0} \delta(t)$ where $h_{c}(t)=h_{c}(-t)^{T} \in \mathbf{L}_{1}^{m \times m}(-\infty, \infty)$ and $h_{0}=h_{0}^{T} \in \mathbf{R}^{m \times m}$. The transfer function $\widehat{\Psi}(s)$ of $\Psi \in$ $S_{A}^{m \times m}$ satisfies $\widehat{\Psi}(s)=\widehat{\Psi}(-s)^{T}$ in its domain of definition, which includes the imaginary axis. It is thus self-adjoint and will be used to define quadratic forms. Similarly, $\mathcal{A}_{d}$ denotes the bounded LTI operators on $l_{2}(0, \infty)$ defined by the convolution in Eq. 3 with an impulse response function satisfying $\sum_{k=0}^{\infty}\left|h_{k}\right|<\infty$. Similarly, $S_{\mathcal{A}_{d}}^{n \times n}$ denotes the LTI bounded operators on $l_{2}^{n}(-\infty, \infty)$ defined by impulse response functions with $h_{k}=h_{-k}^{T}$ and $\sum_{k=-\infty}^{\infty}\left|h_{k}\right|<\infty$. Any transfer function $\widehat{\Psi}(z)$ of $\Psi \in S_{\mathcal{A}_{d}}^{m \times m}$ satisfies $\widehat{\Psi}(z)=$ $\widehat{\Psi}(-z)^{T}$ and is thus self-adjoint. We use the notation $\mathcal{A}$ and $S_{\mathcal{A}}^{n \times n}$ to denote an LTI operator that could be either continuous or discrete time. A causal LTI operator is called stable if its transfer function is analytic in $\Omega$. In particular, $\mathcal{A}$ consists of stable LTI operators.

The diagonal augmentation is defined as

$$
\operatorname{daug}(A, B)=\left[\begin{array}{cccc}
A_{11} & 0 & A_{12} & 0 \\
0 & B_{11} & 0 & B_{12} \\
A_{21} & 0 & A_{22} & 0 \\
0 & B_{21} & 0 & B_{22}
\end{array}\right] .
$$

\section{A model of Heterogeneous Networks}

Our model for a heterogeneous multi-agents network is given in the following system, depicted in Fig. 1

$$
\left\{\begin{array}{l}
{\left[\begin{array}{l}
\boldsymbol{y} \\
\boldsymbol{v}
\end{array}\right]=\left[\begin{array}{ccc}
H_{u y} & H_{r y} & H_{w y} \\
H_{u v} & 0 & H_{w v}
\end{array}\right]\left[\begin{array}{l}
\boldsymbol{u} \\
\boldsymbol{r} \\
\boldsymbol{w}
\end{array}\right]=H\left[\begin{array}{c}
\boldsymbol{u} \\
\boldsymbol{r} \\
\boldsymbol{w}
\end{array}\right],} \\
\boldsymbol{w}=\Delta(\boldsymbol{v}), \\
\boldsymbol{u}=\Gamma(\boldsymbol{y}) .
\end{array}\right.
$$

All the variables are $N$-dimensional vectors, one for each agent. In our model each agent is represented as a common LTI system, so that all the block-entries of $H$ are diagonal and we can write

$$
H=\left[\begin{array}{ccc}
h_{u v} & h_{r y} & h_{w y} \\
h_{u v} & 0 & h_{v v}
\end{array}\right] \otimes I_{N} .
$$

The agent $k$ is characterized by an output $y_{k}$ whose dynamics is given by

$$
y_{k}=h_{u y} u_{k}+h_{r y} r_{k}+h_{w y} w_{k} .
$$

Here $u_{k}$ is the interconnection input, produced according to

$$
u_{k}(t)=\Gamma_{k}(t, \boldsymbol{y}(t)),
$$

where $\Gamma_{k}$ is a bounded memoryless operator $\mathcal{H}_{e}^{N} \rightarrow \mathcal{H}_{e}$ whose structure is related to the communication graph $\mathcal{G}=$ $(V, \mathcal{E})$. In particular, $(j, k) \in \mathcal{E}$ if and only if $\Gamma_{k}$ depends explicitly on $y_{j}$, namely if $k$ is allowed to use the information coming from $j$. The signal $r_{k}$ is called the external input and it can be used, for example, to impose initial conditions under some assumptions on the system. We will always assume that $\|\boldsymbol{r}\|_{2}<\infty$. The internal input $w_{k}$ is an internal signal which is used to model the perturbations of the agents, and it is produced according to $w_{k}=\Delta_{k}\left(v_{k}\right)$, while the internal output $v_{k}$ evolves according to

$$
v_{k}=h_{u v} u_{k}+h_{w v} w_{k} .
$$

The operator $\Delta$ will be always assumed to be diagonal, since the perturbations at different agents are assumed to be "independent" of each other, and even of different nature. For example, $\Delta_{k}$ could be a LTI SISO system while $\Delta_{j}$ could be a memoryless nonlinear operator. If $\Delta(\boldsymbol{v}) \equiv 0$, namely if no perturbation exists, the system evolves according to its nominal behavior, and we have a homogeneous network. The following assumptions are technical conditions that normally are non-restrictive in applications.

Assumption 2.1: The transfer functions representing the operators $h_{u y}, h_{r y}$ and $h_{w y}$ are such that

$$
\hat{h}_{* y}=\frac{b_{* y}}{a} \hat{f}_{* y}, \quad * \in\{u, r, w\},
$$

where $\hat{f}_{* y}$ is the transfer function corresponding to $f_{* y} \in \mathcal{A}$, $b_{* y}$ is a stable polynomial and

$$
a(s)=\prod_{k=1}^{m}\left(s-s_{k}\right)^{\rho_{k}}
$$

where $s_{k} \in \Omega$ such that $\operatorname{deg}\left(b_{* y}\right)<\operatorname{deg}(a)$. We finally assume that $\hat{f}_{* y}$ has no zeros in $\Omega$. This means that the systems are strictly proper, has no unstable zeros, and share the same unstable poles. 


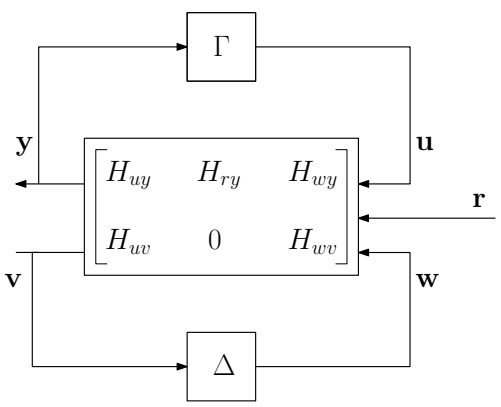

Fig. 1. The system under consideration.

Such assumptions are satisfied in many cases of interest such as the higher order consensus problem with minimum phase nominal system.

Example 2.1: The homogeneous consensus network that was presented in the introduction can be expressed in our framework as

$$
\left\{\begin{array}{l}
{\left[\begin{array}{l}
\boldsymbol{y} \\
\boldsymbol{v}
\end{array}\right]=\left[\begin{array}{ccc}
\frac{z^{-1}}{1-z^{-1}} & \frac{z^{-1}}{1-z^{-1}} & \frac{z^{-1}}{1-z^{-1}} \\
I & 0 & 0
\end{array}\right]\left[\begin{array}{l}
\boldsymbol{u} \\
\boldsymbol{r} \\
\boldsymbol{w}
\end{array}\right]} \\
\boldsymbol{w}=\Delta(\boldsymbol{v}) \\
\boldsymbol{u}=\Gamma_{0} \boldsymbol{y}
\end{array}\right.
$$

where thus $h_{u v}=1, h_{w v}=0$ and $h_{u y}=h_{r y}=h_{y}=$ $\frac{z^{-1}}{1-z^{-1}}, \Gamma_{0}=-(I-P)$ and $\Delta \equiv 0$. If $\Delta \neq 0$ then we have a perturbed consensus network, while the higher order consensus network is obtained simply substituting for $\frac{z^{-1}}{1-z^{-1}}$ a generic transfer function $N_{0}\left(z^{-1}\right)$, or $N_{0}(s)$ in continuoustime. Due to the simplicity of this system, we will often reduce it to $^{2}$

$$
\left\{\begin{array}{l}
y_{k}=N_{0}(I+\Delta) \boldsymbol{u}+N_{0} \boldsymbol{r} \\
\boldsymbol{u}=\Gamma(t, \boldsymbol{y})
\end{array}\right.
$$

which is depicted in Fig. 2, where the operator $\Gamma$ can be more general than the simple multiplication by a constant matrix.

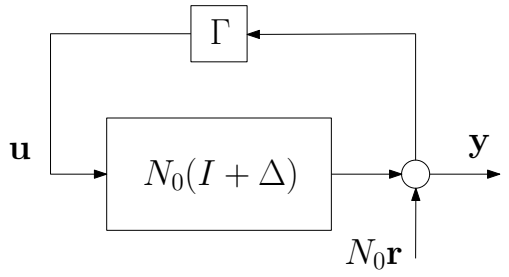

Fig. 2. Higher order perturbed consensus network.

\section{SYNCHRONIZATION OVER HETEROGENEOUS NETWORKS}

In this section we provide the basic tool to prove synchronization in the sense of the following definition.

Definition 3.1: Consider the system in Eq. 4 and a subspace $\mathcal{Z} \subset \mathbb{R}^{N}$. Let $\boldsymbol{y}^{\perp}=\mathcal{P}_{\mathcal{Z}^{\perp}} \boldsymbol{y}$ be the projection of $\boldsymbol{y}$ onto the orthogonal complement of $\mathcal{Z}$. Let $\mathcal{M}: \mathcal{H}_{e}^{N} \rightarrow \mathcal{H}_{e}^{N}$ denote the causal map representing the closed loop system

\footnotetext{
${ }^{2}$ Here $\Delta \boldsymbol{u}$ should be read $\Delta(\boldsymbol{u})$.
}

$\boldsymbol{y}^{\perp}=\mathcal{M}(\boldsymbol{r})$. We say that the system synchronizes to $\mathcal{Z}$ if $\|\mathcal{M}\|_{\mathcal{H}_{e}^{N} \rightarrow \mathcal{H}_{e}^{N}}<\infty$.

This notion of synchronization implies under the assumption $\boldsymbol{r} \in \mathcal{H}$, that $\boldsymbol{y}$ asymptotically converges to $\mathcal{Z}$, and this is why we call it synchronization subspace. Typically $\mathcal{Z}=\operatorname{span}\{\mathbf{1}\}$ in which the term synchronization recovers its usual meaning that the differences among the components of $\boldsymbol{y}$ to converge to zero.

We will take the following steps toward our main synchronization result. First we reduce the dimension of the system by projecting down to the orthogonal complement of $\mathcal{Z}$. Then we perform a loop transformation to stabilize the linear part of the system. The main result will then follow by an application of the IQC theorem in [11].

\section{Projection onto $\mathcal{Z}^{\perp}$}

The following assumption is imposed on the operator $\Gamma$.

Assumption 3.1: The synchronization subspace $\mathcal{Z}$ is the right and left kernel of the memoryless operator $\Gamma$. Namely, if $\boldsymbol{z} \in \mathcal{Z}$, then $\Gamma(t, \boldsymbol{z})=0$ and $\boldsymbol{z}^{*} \Gamma(t, \boldsymbol{v})=0, \forall t \geq 0, \forall \boldsymbol{v} \in$ $\mathcal{H}^{N}$.

Let $Z$ be a matrix whose columns form an orthonormal basis for $\mathcal{Z}$, and $V$ be any orthonormal complement to it, i.e.

$$
Z^{*} Z=I_{p}, V^{*} V=I_{N-p}, V^{*} Z=0, V V^{*}+Z Z^{*}=I_{N}
$$

where $p=\operatorname{dim} \mathcal{Z}$, and where $V V^{*}$ and $Z Z^{*}$ are two projectors respectively onto $\mathcal{Z}^{\perp}$ and $\mathcal{Z}$. Assumption 3.1 can be expressed by means of the constraints

$$
\Gamma(t, \boldsymbol{y})=\Gamma\left(t, V V^{*} \boldsymbol{y}\right), \Gamma(t, \boldsymbol{y})=V V^{*} \Gamma(t, \boldsymbol{y}) .
$$

The first step consists in redefining $\boldsymbol{y}_{\perp}=V^{*} \boldsymbol{y} \in \mathbb{R}^{N-p}$, and the same for $\boldsymbol{u}, \boldsymbol{r}, \boldsymbol{w}, \boldsymbol{v}, \Gamma_{\perp}\left(\boldsymbol{y}_{\perp}\right)=V^{*} \Gamma\left(V \boldsymbol{y}_{\perp}\right)$ and $\Delta_{\perp}\left(\boldsymbol{v}_{\perp}\right)=V^{*} \Delta(V \boldsymbol{y})$. Once this is done, simple computations allow us to conclude that these "projected" variables evolve according to the reduced-dimension system

$$
\left\{\begin{array}{l}
{\left[\begin{array}{l}
\boldsymbol{y}_{\perp} \\
\boldsymbol{v}_{\perp}
\end{array}\right]=H\left[\begin{array}{l}
\boldsymbol{u}_{\perp} \\
\boldsymbol{r}_{\perp} \\
\boldsymbol{w}_{\perp}
\end{array}\right]} \\
\boldsymbol{w}_{\perp}=\Delta_{\perp}\left(\boldsymbol{v}_{\perp}\right) \\
\boldsymbol{u}_{\perp}=\Gamma_{\perp}\left(\boldsymbol{y}_{\perp}\right)
\end{array}\right.
$$

It is worth to notice that the diagonal structure of the linear part $H$ has been maintained after the dimension reduction at the price that the diagonal structure of the perturbation is lost. As we will see, this does not pose any problem.

Remark 3.1: We will assume from now on $H_{w v}=0$, namely we suppress the dependence of the internal output from the internal input. Under some assumptions, this can be done without loss of generality, and this will be the case in all the applications we have in mind.

\section{Loop transformation}

The second step of our approach consists in performing a loop transformation to stabilize the linear part. This is done by means of a matrix

$$
Q=\left[\begin{array}{cc}
0 & Q_{12} \\
Q_{21} & Q_{22}
\end{array}\right]
$$


and by defining the operator $\Gamma_{Q}{ }^{3}$ via the upper linear fractional transformation of $\Gamma_{\perp}, \Gamma_{\perp}=\mathcal{F}_{u}\left(Q, \Gamma_{Q}\right)$, namely

$$
\boldsymbol{u}_{\perp}=\Gamma_{\perp}\left(\boldsymbol{y}_{\perp}\right) \Longrightarrow\left\{\begin{array}{l}
\boldsymbol{u}_{Q}=\Gamma_{Q} \boldsymbol{y}_{Q} \\
{\left[\begin{array}{l}
\boldsymbol{y}_{Q} \\
\boldsymbol{u}_{\perp}
\end{array}\right]=Q\left[\begin{array}{l}
\boldsymbol{u}_{Q} \\
\boldsymbol{y}_{\perp}
\end{array}\right]}
\end{array}\right.
$$

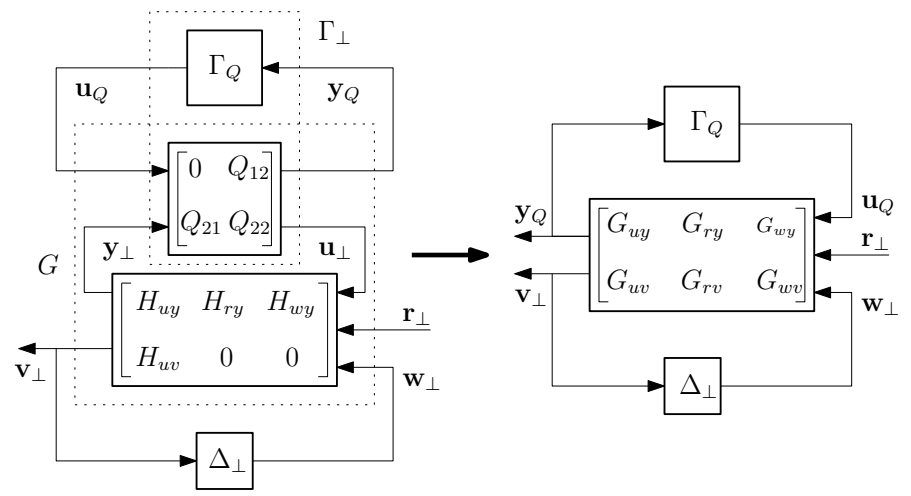

Fig. 3. Loop transformation of the system. The upper linear fractional system $\mathcal{F}_{u}\left(Q, \Gamma_{Q}\right)$ has been substituted for $\Gamma_{\perp}$. The Redheffer star product of $Q$ and the linear and unstable part, $H$, is employed in order to obtain a matrix of stable transfer functions $G$.

This operation is depicted in Fig. 3, in which $\mathcal{F}_{u}\left(Q, \Gamma_{Q}\right)$ is substituted for $\Gamma_{\perp}$. Once this is done, we consider the interconnection of the linear part $H$ and the matrix $Q$, obtaining the matrix of transfer functions

$$
G=Q \star H=\left[\begin{array}{lll}
G_{u y} & G_{r y} & G_{w y} \\
G_{u v} & G_{r v} & G_{w v}
\end{array}\right]
$$

where the star product defines

$$
\begin{aligned}
& G_{u y}=h_{u y} Q_{12}\left(I-Q_{22} h_{u y}\right)^{-1} Q_{21} \\
& {\left[\begin{array}{ll}
G_{r y} & G_{w y}
\end{array}\right]=Q_{12}\left(I-Q_{22} h_{u y}\right)^{-1}\left[\begin{array}{ll}
h_{r y} & h_{w y}
\end{array}\right]} \\
& G_{u v}=h_{u v}\left(I-Q_{22} h_{u y}\right)^{-1} Q_{21} \\
& {\left[\begin{array}{ll}
G_{r v} & G_{w v}
\end{array}\right]=h_{u v} Q_{22}\left(I-Q_{22} h_{u y}\right)^{-1}\left[\begin{array}{ll}
h_{r y} & h_{w y}
\end{array}\right]}
\end{aligned}
$$

If $G$ is stable, we have thus achieved the goal of this section, and this will be an assumption from now on.

Assumption 3.2: The matrix $Q \in \mathbb{R}^{2 N \times 2 N}$ is chosen in such a way that all the entries of the matrix of transfer functions $G$ in Eq. 8 are stable.

\section{The synchronization criterion}

The main result will follow by an application of the IQC theorem from [11] on the transformed system. Stability and Integral Quadratic Constraint are defined as follows.

Definition 3.2: The interconnection $\left[G, \operatorname{diag}\left(\Gamma_{Q}, \Delta_{\perp}\right)\right]$ in Fig. 4 is called stable if there exists $c>0$ such that

$$
\left\|y_{Q}\right\|^{2}+\left\|v_{\perp}\right\|^{2} \leq c\left\|r_{\perp}\right\|^{2}
$$

for all $r_{\perp} \in \mathcal{H}^{n-1}$.

\footnotetext{
${ }^{3}$ We will often suppress the arguments of $\Gamma(t, \boldsymbol{y})$, and of $\Gamma_{Q}$ and $\Gamma_{\perp}$, for sake of notation.
}

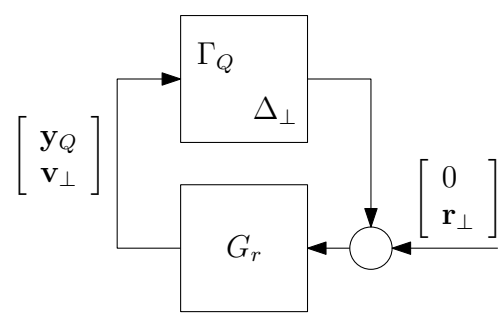

Fig. 4. Feedback system on which we apply the IQC theorem. Here $G_{r}$ is a matrix of stable transfer functions and in order to prove stability we have to provide IQC characterizations for both the operators $\Gamma_{Q}$ and $\Delta$.

Definition 3.3 (IQC): Let $\Pi \in S_{\mathcal{A}}^{2 m \times 2 m}$. Then a bounded causal operator $\Delta: \mathcal{H}_{e}^{m} \rightarrow \mathcal{H}_{e}^{m}$ is said to satisfy the IQC defined by $\Pi(\Delta \in I Q C(\Pi))$ if

$$
\left\langle\left[\begin{array}{c}
\Delta(w) \\
w
\end{array}\right], \Pi\left[\begin{array}{c}
\Delta(w) \\
w
\end{array}\right]\right\rangle \leq 0, \forall w \in \mathcal{H} .
$$

We are now ready to state our main result.

Theorem 3.1: Assume that the operator $\Gamma$ respects Assumption 3.1 and that there exists a matrix $Q \in \mathbb{R}^{2 N \times 2 N}$ which respects Assumption 3.2. Assume moreover that

i) there exists continuous (in the norm topology) parametrizations $\Gamma_{Q}(\tau)$ and $\Delta_{\perp}(\tau)$ such that $\Gamma_{Q}(1)=$ $\Gamma_{Q}, \Delta_{\perp}(1)=\Delta_{\perp}$ and such that the nominal interconnection $\left[G, \operatorname{diag}\left(\Gamma_{Q}(0), \Delta_{\perp}(0)\right)\right]$ is stable,

ii) there exists bounded self-adjoint linear operators $\Pi_{\Gamma_{Q}}$ and $\Pi_{\Delta_{\perp}}$ such that

(a) $\Gamma_{Q}(\tau) \in \operatorname{IQC}\left(\Pi_{\Gamma_{Q}}\right), \tau \in[0,1]$,

(b) $\Delta_{\perp}(\tau) \in I Q C\left(\Pi_{\Delta_{\perp}}\right), \tau \in[0,1]$,

iii)

$$
\left[\begin{array}{c}
I \\
G_{r}
\end{array}\right]^{*} \operatorname{daug}\left(\Pi_{\Gamma_{Q}}, \Pi_{\Delta_{\perp}}\right)\left[\begin{array}{c}
I \\
G_{r}
\end{array}\right]>0
$$

where

$$
G_{r}=\left[\begin{array}{ll}
G_{u y} & G_{w y} \\
G_{u v} & G_{w v}
\end{array}\right],
$$

iv) $h_{u v}, h_{u v}^{-1} \in \mathcal{A}, Q_{12}, Q_{12}^{-1} \in \mathbb{R}^{N \times N}$.

Then the network in Eq. 4 synchronizes to the subspace $\mathcal{Z}$ in the sense of Definition 3.1

Remark 3.2: The theorem ensures that $\boldsymbol{y}_{\perp}$ belongs to $\mathcal{H}^{N-p}$. In discrete-time this is enough for $\boldsymbol{y}_{\perp} \rightarrow 0$, while in continuous-time we need to impose in addition that $G_{u y}$, $G_{r y}$ and $G_{w y}$ are strictly proper. However, this is true by our assumptions on $h_{* y}, * \in\{u, r, w\}$, and hence $\boldsymbol{y} \rightarrow \mathcal{Z}$, namely the network synchronizes in the usual sense.

Remark 3.3: In general, multipliers able to tightly catch the characteristics of the operators $\Gamma_{Q}$ and $\Delta_{\perp}$ allow less conservative criteria. On the contrary, badly chosen ones could result in conditions which cannot be satisfied, and thus the criterion turns out to be useless.

\section{SPECTRAL DECOMPOSITION OF THE INTERCONNECTION MATRIX $\Gamma_{0}$}

In this section we particularize the previous result for the simplest choice for the interconnection operator $\Gamma(t, \boldsymbol{y})$, namely the multiplication by a constant matrix

$$
\Gamma(t, \boldsymbol{t})=\Gamma_{0} \boldsymbol{y}
$$

and we impose the following Assumption on $\Gamma_{0}$. 
Assumption 4.1: The matrix $\Gamma_{0}$

i) respects Assumption 3.1

iia) is normal, namely such that $\Gamma_{0} \Gamma_{0}^{*}=\Gamma_{0}^{*} \Gamma_{0}$

iib) is such that the transfer functions $\frac{h_{r y}}{1-\lambda_{k} h_{u y}}$ are stable for any non-zero eigenvalue $\lambda_{k}$ of $\Gamma_{0}$.

Normality of $\Gamma_{0}$ implies that

$$
\Gamma_{0}=[Z V]\left[\begin{array}{cc}
0_{p} & 0 \\
0 & \Gamma_{0 \perp}
\end{array}\right]\left[\begin{array}{l}
Z^{*} \\
V^{*}
\end{array}\right]=V^{*} \Gamma_{0} V,
$$

where the columns of $Z$ are an orthonormal basis of $\mathcal{Z}=$ $\operatorname{ker} \Gamma_{0}$, those of $V$ are an orthonormal basis of $\mathcal{Z}^{\perp}$, and $\Gamma_{0 \perp}=\operatorname{diag}\left(\lambda_{p+1}, \ldots, \lambda_{N}\right) \in \mathbb{R}^{N-p \times N-p}$ is a diagonal matrix whose $(k, k)$-th entry is the $k$-th non-zero eigenvalue of $\Gamma_{0}$. The following result can be proved using Theorem 3.1 once we choose for the loop transformation the following

$$
Q_{12}=I_{N-1}, Q_{21}=I_{N-1}, Q_{22}=\Gamma_{0 \perp} .
$$

Corollary 4.1: Consider the system in Eq. 4 in which $\Gamma(t, \boldsymbol{y})=\Gamma_{0} \boldsymbol{y}$, where $\Gamma_{0}$ respects Assumption 4.1.

Assume there exists a multiplier $\pi_{\Delta} \in S_{\mathcal{A}_{d}}^{2 \times 2}$ such that $\pi_{\Delta, 11} \geq 0$ and $\pi_{\Delta, 22} \leq 0, \Delta_{k} \in I Q C\left(\pi_{\Delta}\right)$ and

$$
\left[\begin{array}{c}
I \\
\frac{\lambda_{k} h_{r y}}{1-\lambda_{k} h_{u y}}
\end{array}\right]^{*} \pi_{\Delta}\left[\begin{array}{c}
I \\
\frac{\lambda_{k} h_{r y}}{1-\lambda_{k} h_{u y}}
\end{array}\right]>0,
$$

for any nonzero eigenvalue $\lambda_{k}, k=1, \ldots, N-p$ of $\Gamma_{0}$. Assume moreover that $h_{u v}, h_{u v}^{-1} \in \mathcal{A}$. Then the system synchronizes to the subspace $\mathcal{Z}$.

\section{A. Quasi-saturation in the interconnection inputs}

In this section we consider the higher order consensus system which we have described in Example 2.1

$$
\left\{\begin{array}{l}
\boldsymbol{y}=N_{0}(1+\Delta) \boldsymbol{u}+N_{0} \boldsymbol{r} \\
\boldsymbol{u}=\Gamma_{0} \boldsymbol{u}
\end{array}\right.
$$

The input to the $k$-th agent at time $t$ is given by $u_{k}+$ $\Delta_{k}\left(u_{k}(t)\right)$ and we assume that $\Delta_{k}(u)=0$ if $|u| \leq u_{t h}$, where $u_{t h}$ is a certain threshold value, and that in general it satisfies the slope restriction

$$
-\alpha_{\min } \leq \frac{\Delta_{k}\left(x_{1}\right)-\Delta_{k}\left(x_{2}\right)}{x_{1}-x_{2}} \leq 0 .
$$

By making use of such an operator we can model quasisaturation of the input, in which the interconnection input $u_{k}(t)$ is used if it is small enough in absolute value, while if it is too large it is underestimated. If $\alpha_{\min }=0$ then the effect of $\Delta_{k}$ disappears, and we keep $\alpha_{\min }<1$ in order to avoid the pure saturation of the input, which in general could prevent our notion of synchronization. An example of what can be expressed using this $\Delta$ is shown in Fig. 5 .

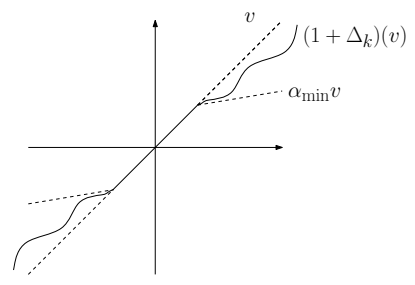

Fig. 5. An example of quasi-saturation.
A first, simple multiplier is the sector-condition multiplier

$$
\pi_{\Delta, C}=\left[\begin{array}{cc}
2 & \alpha_{\min } \\
\alpha_{\min } & 0
\end{array}\right]
$$

which, however, offers too conservative results. We will combine it with the Zames-Falb multiplier, see e.g. [12],

$$
\pi_{\Delta, Z F}(j \omega)=\left[\begin{array}{cc}
2 \operatorname{Re}\left(\frac{-j \omega}{1-j \omega / \tau}\right) & \alpha_{\min } \frac{-j \omega}{1-j \omega / \tau} \\
\alpha_{\min } \frac{j \omega}{1+j \omega / \tau} & 0
\end{array}\right] .
$$

for which $\Delta_{k} \in I Q C\left(\pi_{\Delta, Z F}\right)$ thanks to the slope condition. If we choose $\tau$ large enough, this bounded multiplier approximates, at sufficiently low frequency, as $\pi_{\Delta, Z F}(j \omega) \approx$ $\alpha_{\min } \pi_{\Delta, P}(j \omega)$, where the Popov multiplier is

$$
\pi_{\Delta, P}(j \omega)=\left[\begin{array}{cc}
0 & -j \omega \\
j \omega & 0
\end{array}\right] .
$$

Hence, at low frequencies the Zames-Falb multiplier recovers the Popov one.

Our choice for the multiplier to be used is a linear combination of the two

$\pi_{\Delta, C}+\lambda \alpha_{\min } \pi_{\Delta, P}=\left[\begin{array}{cc}2 & \alpha_{\min }(1-j \lambda \omega) \\ \alpha_{\min }(1+j \lambda \omega) & 0\end{array}\right]$.

We can invoke Corollary 4.1 to immediately prove the following result.

Corollary 4.2: Consider the system in Eq. 11 where $\Delta_{k}$ satisfies Eq. 12 and $\Gamma_{0}$ satisfies Assumption 4.1. Then the system synchronizes if there exists $\lambda \in \mathbb{R}$ such that

$$
\left[\begin{array}{c}
1 \\
\frac{\lambda_{k} N_{0}}{1-\lambda_{k} N_{0}}
\end{array}\right]^{*}\left(\pi_{\Delta, C}+\lambda \pi_{\Delta, P}\right)\left[\begin{array}{c}
1 \\
\frac{\lambda_{k} N_{0}}{1-\lambda_{k} N_{0}}
\end{array}\right]>0,
$$

where $\pi_{\Delta, C}$ and $\pi_{\Delta, P}$ are defined above.

This inequality can easily be checked graphically using a Popov plot. Define $G_{r}=\frac{\lambda_{k} N_{0}}{1-\lambda_{k} N_{0}}$ and

$$
\mathcal{P}=\left\{z: z=\operatorname{Re} G_{r}(j \omega)-j \omega \operatorname{Im} G_{r}(j \omega)\right\} .
$$

The system synchronizes if $\mathcal{P}$ entirely lies on the right to the line with slope $\frac{1}{\lambda}$ and crossing the $x$-axis in the point $-\frac{1}{\alpha_{\min }}$. Is is worth to notice that if $\alpha_{\min } \rightarrow 0$, namely if $\Delta_{k} \rightarrow 0$, then the Popov criterion is always satisfied, and this is clear since the nominal interconnection is stable.

Remark 4.1: Corollary 4.2 and the graphical criterion are stated and verified using the Popov multiplier instead of the Zames-Falb multiplier. As we have noticed above, the former is a good approximation of the latter at low frequencies, so in this range the two criteria essentially coincide. One has then to be sure that at high frequencies in which the correct multiplier is $\pi_{\triangle, Z F}$, the inequality in Eq. 14 is satisfied. This holds, for example, if $\tau$ is large, we are in continuous-time and $N_{0}(s)$ is a strictly proper transfer function.

\section{B. Clocks Synchronization}

In this section we will apply our result to the continuoustime version of the clock model presented in [13]. A clock is modeled as a double-integrator in which the first state is the relative time given by the clock and the second is the instantaneous skew of the clock. The model in state space is

$$
\left\{\begin{array}{l}
\dot{x}_{k}(t)=\left[\begin{array}{ll}
0 & q \\
0 & 0
\end{array}\right] x_{k}(t)+F u_{k}(t), \\
y_{k}(t)=\left[\begin{array}{ll}
1 & 0
\end{array}\right] x_{k}(t)
\end{array}\right.
$$


A steady assumption will be that the parameter $q$ is shared among all the clocks. This is a rather strong assumption which is done for simplicity only. The control matrix $F=$ $\left[f_{1}, f_{2}\right]^{T}$ is assumed to be the same for all the clocks, and $u_{k}$ is the $k$-th component of $\boldsymbol{u}(t)=(I+\Delta)\left(\Gamma_{0} \boldsymbol{y}(t)\right)$.

To give a numerical example, consider a network of 9 clocks and set $q=1$ and $f_{1}=1.7, f_{2}=1$, thus the transfer function modeling the clocks is $N_{0}(s)=\frac{f_{1} s+f_{2} q}{s^{2}}=\frac{1.7 s+1}{s^{2}}$. We set as interconnection matrix $\Gamma_{0}=-I_{N}^{s^{2}}+0.15\left(\mathcal{C}_{N}+\right.$ $\left.\mathcal{C}_{N}^{-1}\right)+0.30 \mathcal{C}_{N}^{5}+0.40 \mathcal{C}_{N}^{-5}$, where $\mathcal{C}_{N}$ is the $N \times N$ circulant matrix whose first row is entirely zero apart from $\left[\mathcal{C}_{N}\right]_{12}=1$. It can be checked that $\frac{N_{0}(s)}{1-\lambda_{k} N_{0}(s)}$ is a stable transfer function for all $k$.

We assume thus that the systems are all equal, except that they all quasi-saturate their inputs as

$$
\begin{array}{ll}
\left(1+\Delta_{k}\right)\left(u_{k}(t)\right)= & \\
\qquad \begin{cases}u_{k}(t), & \left|u_{k}(t)\right| \leq u_{t h} \\
u_{t h}+\alpha_{k}\left(u_{k}(t)-\operatorname{sgn}\left(u_{k}(t)\right) u_{t h}\right), & \left|u_{k}(t)\right|>u_{t h}\end{cases}
\end{array}
$$

where $\alpha_{k} \in[0.2,1], \forall k$.

To prove the synchronization of this network we can use the multiplier $\pi_{\Delta}$ in Eq. 13 with $\lambda=3$, and to see that the IQC is satisfied one can use the Popov plot, which is presented in Fig. 7.

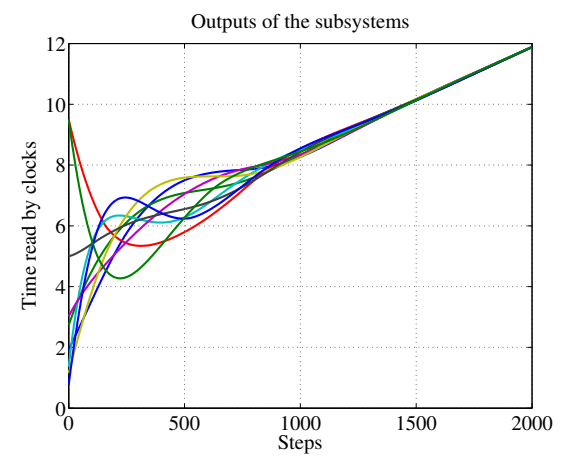

Fig. 6. Clock synchronization with quasi-saturation. A typical trajectory of the outputs.

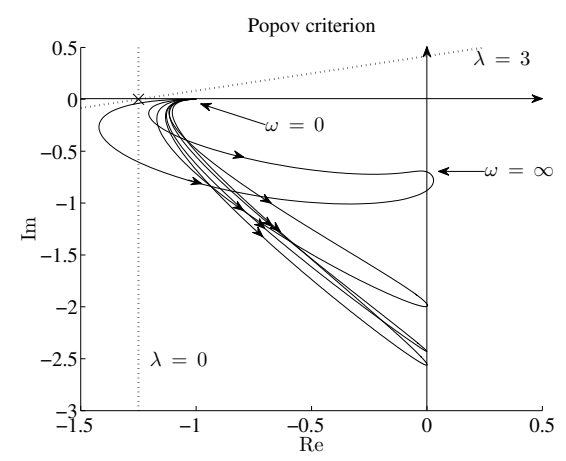

Fig. 7. The Popov plot in the case of quasi saturation. The big cross marks the point $-\frac{1}{\alpha_{\min }}+j 0$.

\section{A non-normal $\Gamma_{0}$ example}

In this section the interconnection matrix $\Gamma_{0}$ is not longer assumed to be normal. Instead, we assume $\Gamma_{0}=-\nu L$ where $\nu>0$ is a real number and $L$ is a reversible weighted Laplacian of the communication graph $\mathcal{G}$. Being a Laplacian means that $L_{j i} \leq 0 \Longleftrightarrow(i, j), i \neq j$, is an edge of the graph, and that $L \mathbf{1}=0$. A Laplacian can be obtained as $L=I-P$ where $P$ is a non-negative row-stochastic matrix associated with the communication graph. By PerronFrobenius theorem the kernel of $L$ is only spanned by 1 if $P$ is primitive, for which a sufficient condition is presented in the following proposition, adapted from [1].

Proposition 4.1: Consider a row-stochastic matrix $P$ associated to the directed graph $\mathcal{G}$. Assume that $\mathcal{G}$ has all the self-loops, namely that $P_{i i}>0, \forall i=1, \ldots, N$, and that $\mathcal{G}$ has a spanning tree. Then the matrix $P$ is primitive.

Reversibility of $\Gamma_{0}$ means that there exists a diagonal matrix $D$ such that

$$
D \Gamma_{0}=\Gamma_{0}^{T} D,
$$

which, if it exists, can be obtained as $D=\operatorname{diag}(\boldsymbol{\pi})$ where $\pi^{T} \Gamma_{0}=0$. Notice that in the special case $D=\frac{1}{N} I_{N}$, the matrix $\Gamma_{0}$ is symmetric so we fall in the normal case.

Assume now that $\pi_{i}>0, \forall i=1, \ldots, N$, so that we can write $D^{1 / 2}$ and $D^{-1 / 2}$, and define the matrix

$$
R_{0}=D^{1 / 2} \Gamma_{0} D^{-1 / 2} \text {. }
$$

Using Eq. 16 it is trivial to see that $R_{0}$ is symmetric, and this proves that the eigenvalues of a reversible $\Gamma_{0}$ are real, since $R_{0}$ and $\Gamma_{0}$ are similar. Since $R_{0}$ is symmetric, it is also normal, and its right kernel is $\operatorname{span}\left\{D^{1 / 2} \mathbf{1}\right\}$.

Consider the higher order consensus system

$$
\left\{\begin{array}{l}
\boldsymbol{y}=N_{0}(I+\Delta) \boldsymbol{u}+N_{0} \boldsymbol{r} \\
\boldsymbol{u}=\Gamma_{0} \boldsymbol{y}
\end{array} .\right.
$$

We can perform a multiplier transformation defining $\overline{\boldsymbol{y}}=$ $D^{1 / 2} \boldsymbol{y}, \overline{\boldsymbol{u}}=D^{1 / 2} \boldsymbol{u}, \overline{\boldsymbol{r}}=D^{1 / 2} \boldsymbol{r}$ and $\bar{\Delta}(\overline{\boldsymbol{u}})=$ $D^{1 / 2} \Delta\left(D^{-1 / 2} \overline{\boldsymbol{u}}\right)$ in order to obtain

$$
\left\{\begin{array}{l}
\overline{\boldsymbol{y}}=N_{0}(I+\bar{\Delta}) \overline{\boldsymbol{u}}+N_{0} \overline{\boldsymbol{r}} \\
\overline{\boldsymbol{u}}=R_{0} \overline{\boldsymbol{y}}
\end{array}\right.
$$

where now $R_{0}$ respects the normality assumptions. We can now state the following result.

Proposition 4.2: Consider the system in Eq. 18 where $\Gamma_{0}=-\nu(I-P)$ is reversible with left kernel spanned by $\pi^{T}$. Assume moreover that the transfer functions $\frac{N_{0}}{1-\lambda_{k} N_{0}}$ are stable for any nonzero eigenvalue of $\Gamma_{0}$. Then if there exists a multiplier $\pi_{\Delta} \in S_{\mathcal{A}}^{2 \times 2}$ such that $\pi_{\Delta, 11} \geq 0, \pi_{\Delta, 22} \leq 0$, $\Delta_{k} \in I Q C\left(\pi_{\Delta}\right)$ and

$$
\left[\begin{array}{c}
I \\
\frac{\lambda_{k} N_{0}}{1-\lambda_{k} N_{0}}
\end{array}\right]^{*} \pi_{\Delta}\left[\begin{array}{c}
I \\
\frac{\lambda_{k} N_{0}}{1-\lambda_{k} N_{0}}
\end{array}\right]>0,
$$

for any nonzero eigenvalue $\lambda_{k}, k=1, \ldots, N-p$ of $\Gamma_{0}$. Then the system synchronizes to the subspace $\mathcal{Z}=\operatorname{span}\{\mathbf{1}\}$.

1) Leader following using reversible matrices: The previous paragraph dealt with the case $\pi_{i}>0, \forall i=1, \ldots, N$. In this paragraph we study what happens if some of them are zero in the simple case $\Delta_{k}=0, k=1, \ldots, N$ for sake of simplicity. Assume $V=S_{1} \cup S_{2}, S_{1}=\{1, \ldots, q\}$ and $S_{2}=\{q+1, \ldots, N\}$, and $\pi_{i}>0$ if $i \in S_{1}$ and $\pi_{j}=0$ if $j \in S_{2}$. By reversibility $\Gamma_{0}$ has the structure

$$
\Gamma_{0}=\left[\begin{array}{cc}
\Gamma_{S_{1}} & 0 \\
\Gamma_{S_{12}} & \Gamma_{S 2}
\end{array}\right]
$$


By suitably partitioning the system it is not difficult to see that the agents in $S_{1}$ evolve according to

$$
\left\{\begin{array}{l}
\boldsymbol{y}_{S_{1}}=N_{0} I_{S_{1}} \boldsymbol{u}_{S_{1}}+N_{0} \boldsymbol{r}_{S_{1}}, \\
\boldsymbol{u}_{S_{1}}=\Gamma_{S_{1}} \boldsymbol{y}_{S_{1}}
\end{array}\right.
$$

while the dynamics of those in $S_{2}$ is

$$
\boldsymbol{y}_{S_{2}}=\left(I-\Gamma_{S_{2}} N_{0}\right)^{-1} N_{0}\left(\Gamma_{S_{12}} \boldsymbol{y}_{S_{1}}+\boldsymbol{r}_{S_{2}}\right) \text {. }
$$

Because of the block-lower-triangular structure of $\Gamma_{0}, \Gamma_{S_{1}}$ is minus a Laplacian while the eigenvalues of $\Gamma_{S_{2}}$ are all stable and if the matrix $\Gamma_{0}$ is chosen in such a way that all its nonzero eigenvalues are able to stabilize $N_{0}$, then $\left(I-\Gamma_{S_{2}} N_{0}\right)^{-1} N_{0}$ is a stable matrix of transfer functions. Notice, moreover, that since $\Gamma_{0} \mathbf{1}=0$, we have $\Gamma_{S_{2}}^{-1} \Gamma_{S_{12}} \mathbf{1}=$ -1. Since $r_{S_{2}}$ is a bounded signal and $y_{S_{1}}(t) \rightarrow \alpha(t) \mathbf{1}$, we can conclude that the agents in $S_{1}$ reach asymptotically an agreement and act as leaders, while set $S_{2}$ will follow it, progressively forgetting initial conditions and external inputs. Setting $\boldsymbol{y}_{S_{1}}(t)=V \boldsymbol{y}_{\perp}+\boldsymbol{y}_{\mathbf{1}}$, where $\boldsymbol{y}_{\mathbf{1}}=\alpha(t) \mathbf{1}$, we obtain

$$
\boldsymbol{y}_{S_{2}}=-\left(\Gamma_{S_{2}}^{-1}-N_{0}\right)^{-1} N_{0} \boldsymbol{y}_{1}+\boldsymbol{q}
$$

where $\boldsymbol{q}=\left(I-\Gamma_{S_{2}} N_{0}\right)^{-1} N_{0} \Gamma_{S_{2}}^{-1}\left(\Gamma_{S_{12}} V \boldsymbol{y}_{\perp}+\boldsymbol{r}_{S_{2}}\right) \in$ $\mathcal{H}^{N-q}$. From this one may consider different cases. If $\alpha(t)=$ $\alpha_{0}$ (a constant) and $N_{0}(z)$ has its only unstable pole at $z=1$, then the final value theorem gives

$$
\lim _{t \rightarrow \infty} \boldsymbol{y}_{S_{2}}(t)=-\lim _{z \rightarrow 1}\left(\Gamma_{S_{2}}^{-1}-N_{0}(z)\right)^{-1} N_{0}(z) \alpha_{0} \mathbf{1}=\alpha_{0} \mathbf{1}
$$

Similarly if $\alpha(t)=A \sin \left(\omega_{0} t+\phi_{0}\right)$ and $N_{0}(s)=\frac{1+s}{s^{2}+\omega_{0}^{2}}$, $\boldsymbol{y}_{S_{2}}(t)$ converges to the sinusoid $A \sin \left(\omega_{0} t+\phi_{0}\right)$.

A numerical example: Consider a homogeneous network of $N=8$ oscillators whose nominal dynamics is $N_{0}(s)=$ $\frac{1+s}{s^{2}+\omega_{0}^{2}}$ where $\omega_{0}=1$. The network is divided in two subsets (see Fig. 8), a first set $S_{1}$ in which the agents are interconnected in a circle and communicate via the matrix $\Gamma_{S_{1}}=-I_{4}+0.5 \mathcal{C}_{4}+0.5 \mathcal{C}_{4}^{-1}$, and a second set $S_{2}$ in which the agents are interconnected in a circle via a matrix $\Gamma_{S_{2}}$ and, moreover, can receive information from one of the agents of the set $S_{1}$ via a matrix $\Gamma_{S_{12}}$.

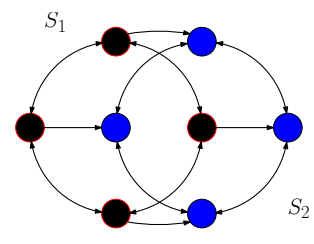

Fig. 8. Leader following for a network of perturbed oscillators. The graph of communication. In black it is depicted the set $S_{1}$ of agents, in blue it is the set $S_{2}$. The agents in $S_{2}$ receive information from $S_{1}$ without replying.

The two $\Gamma_{S_{2}}$ and $\Gamma_{S_{12}}$, apart from the structure, are chosen randomly with the only constraint that $\left[\begin{array}{ll}\Gamma_{S_{12}} & \Gamma_{S_{2}}\end{array}\right] \mathbf{1}=0$. We simulate the system taking random initial conditions, and obtain as a typical trajectory what is depicted in Fig. 9. As it can be seen, the agents in $S_{1}$ agree on a sinusoid of angular frequency $\omega_{0}=1 \mathrm{rad} / \mathrm{sec}$, the "nominal behavior", followed by the agents in $S_{2}$ which forget their initial conditions and slowly converge to the behavior of the former agents.

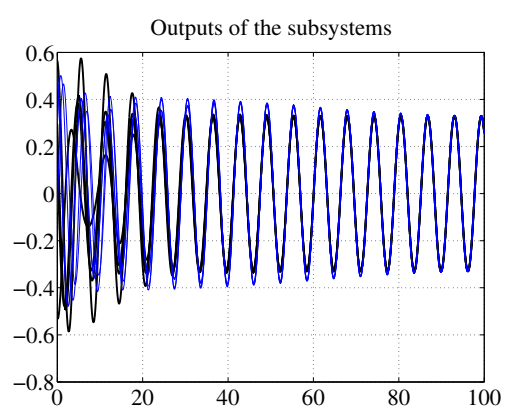

Fig. 9. Leader following for a network of perturbed oscillators. In thick black the trajectory of the leaders, in thin blue the followers.

\section{CONCLUSIONS AND FUTURE WORK}

The paper presents a model for heterogeneous networks of agents modeled by a perturbed nominal linear SISO system and interconnected via a memoryless operator. We have in preparation a second paper in which we rephrase the result in the original variables and we study in detail the case in which each perturbation operator $\Delta_{k}$ is a LTI SISO system, for which a Nyquist criterion can be stated similarly to what we have done in [5]. Future work includes and is not limited to dynamic interconnection operators and time-switching networks.

\section{REFERENCES}

[1] W. Ren and R. Beard, "Consensus seeking in multiagent systems under dynamically changing interaction topologies," IEEE Tran. on Aut. Control, vol. 50, no. 5, May 2005.

[2] R. Olfati-Saber, J. Fax, and R. Murray, "Consensus and cooperation in networked multi-agent systems," Proceedings of the IEEE, vol. 95, no. 1 , jan. 2007.

[3] F. Gantmacher, The theory of matrices. Chelsea publ., 1959.

[4] U. Jönsson and C.-Y. Kao, "A scalable robust stability criterion for systems with heterogeneous lti components," IEEE Tran. on Aut. Control, 2010.

[5] E. Lovisari and U. Jönsson, "A nyquist criterion for synchronization in networks of heterogeneous linear systems," in Proceedings of Workshop on Distributed Estimation and Control in Networked Systems, NecSys'10, September 2010.

[6] I. Lestas and G. Vinnicombe, "Heterogeneity and scalability in group agreement protocols: Beyond small gain and passivity approaches," Automatica, vol. 46, no. 7, 2010.

[7] L. Scardovi, M. Arcak, and E. Sontag, "Synchronization of interconnected systems with applications to biochemical networks: An inputoutput approach," IEEE Tran. on Aut. Control, june 2010.

[8] G.-P. Jiang, W. K.-S. Tang, and G. Chen, "A state-observer-based approach for synchronization in complex dynamical networks," IEEE Trans. on Circuits and Systems, 2006.

[9] U. Jönsson, C.-Y. Kao, and H. Fujioka, "A Popov criterion for networked systems," Systems and Control Letters, 2007.

[10] S. Hara and H. Tanaka, "D -stability and robust stability conditions for lti systems with generalized frequency variables," in Proceedings of 49th Conference on Decision and Control, CDC'10, December 2010.

[11] A. Megretski and A. Rantzer, "System analysis via integral quadratic constraints," Automatic Control, IEEE Transactions on, jun 1997.

[12] G. Zames and P. Falb, "Stability conditions for systems with monotone and slope-restricted nonlinearities," SIAM Journal of Control, vol. 6, no. 1 , pp. 89-108, 1968.

[13] R. Carli, A. Chiuso, L. Schenato, and S. Zampieri, "Distributed synchronization of noisy non-identical double integrators," IEEE Tran. on Aut. Control, 2011. 